\title{
The Parkes Multibeam Blind HI Survey
}

\author{
R.L. Webster, V. Kilborn and J.C. O'Brien
}

School of Physics, University of Melbourne, Parkville, Vic, Australia, 3052

\section{Staveley-Smith}

Australia Telescope National Facility, PO Box 76, Epping, NSW, Australia, 2121

\section{M.E. Putman}

Mount Stromlo and Siding Springs Observatory, Australian National University, Weston Creek PO., ACT, Australia, 2611

\section{G. Banks}

Department of Physics and Astronomy, University of Wales, Cardiff, PO Box 913, Cardiff, CF2 3YB, Wales, UK

\begin{abstract}
A thirteen-beam HI receiver has been constructed for the Parkes radio telescope. When this instrument is used in active scanning mode, it can rapidly survey large areas of sky, with a relatively uniform sensitivity. The Multibeam Working Group, comprising about 30 astronomers from more than a dozen institutions, is undertaking a blind HI survey of the entire southern sky. The status of the survey is described, with some of the first scientific results.
\end{abstract}

\section{The Survey}

Surveys of large areas of sky in $\mathrm{HI}$ are time-consuming, and require either a dedicated telescope (e.g. Dwingeloo Obscured Galaxy Survey (Henning et al. 1998)) or the multiplexing advantage of a multibeam system. The multibeam receiver at Parkes is a purpose-built instrument with 13 receivers, arranged in a hexagonal grid (Staveley-Smith 1997). The beam centres are separated by about two beam widths. With an appropriate orientation, the full array will Nyquist sample as the telescope scans the sky.

Two surveys are underway: the HI All-sky Survey (HIPASS) which will survey the region $\delta<0^{\circ}$ (though a northern extension is being discussed), and a more sensitive Zone-of-Avoidance survey (ZOA), within $5^{\circ}$ of the galactic plane. Details of the HIPASS survey will be described. For a more complete description of the ZOA survey see Henning et al (1998). The HIPASS survey uses active scanning where the telescope is driven at $1^{\circ}$ per minute in declination strips, recording a spectrum every $5 \mathrm{sec}$. Each region of sky is scanned 5 times, with small offsets between each set of scans. Data is band-pass corrected online, and 
both the raw and the preprocessed data are archived (Barnes et al. 1998). On the completion of the full set of scans, data is gridded onto the sky in cubes of $8^{\circ} \times 8^{\circ}$, with $4^{\prime}$ pixels.

In mid-1998, the survey was $\sim 40 \%$ complete. Extensive modelling of the gridding procedure has resulted in a fast robust gridder (Barnes 1998). The survey parameters are given in the Table 1.

Table 1. Survey parameters, updated from Staveley-Smith (1997)

\begin{tabular}{cc}
\hline Parameter & Value \\
\hline \hline Declination range & $\delta<0^{\circ}$ \\
Equivalent Integration time & $500 \mathrm{sec}$ \\
Velocity Range & $13.2 \mathrm{~km} \mathrm{sec}^{-1}$ \\
Channel Resolution & $18.0 \mathrm{~km} \mathrm{sec}^{-1}$ \\
Velocity Resolution & $2-5^{\prime}$ \\
Positional Accuracy $(3 \sigma)$ & $40 \mathrm{mJy} \mathrm{per} \mathrm{beam}^{-1}$ \\
Detection Limit $(3 \sigma)$ & $1.4 \times 10^{6} d_{M p c}^{2} M_{\odot}$ \\
HI Mass Limit $(3 \sigma)$ & $\theta \gtrsim 7^{\prime}$ \\
Angular Resolution & 388 \\
Limiting Column Density $(3 \sigma)$ & $7 \times 10^{17} \mathrm{HI} \mathrm{cm}^{-2}$ per velocity channel \\
Number of Data Cubes & 388 \\
\hline \hline
\end{tabular}

At the present time, galaxy finding is by eye. Cubes are scanned in velocityposition space for regions of enhanced HI emission. This process is both timeconsuming and statistically unquantifiable. The Multibeam Working Group is investigating automated galaxy-finding methods which will increase the speed of this process and provide quantifiable detection limits. Nearly all galaxy detections are unresolved on the sky. Thus we are searching for point sources which are distributed in velocity space. Our most promising techniques are based on wavelet transforms, using a point source profile with variable filtering in velocity space. The current flux limit for detection by eye is $\sim 4 \mathrm{Jy} \mathrm{km} \mathrm{sec}^{-1}$, which is equivalent to $\sim 9 \sigma$ for a velocity width of $\sim 70 \mathrm{~km} \mathrm{sec}^{-1}$. The principle issue for the galaxy finder is to efficiently find galaxies in data with non-gaussian noise. However we are also investigating new methods of filtering noise from the data. An automated finder will provide a powerful tool with which to explore the data cubes and will be necessary before an all-sky catalogue of galaxies can be published.

In order to fully realise the scientific program associated with the survey, a complimentary followup program is required. Synthesised images of selected HI detections are being obtained at the ATCA. These images not only provide more accurate positions for optical identifications, but also resolve the HIPASS detections both spatially and in velocity (Kilborn et al. 1998). Optical $B$ and $R$-band images are being obtained on 1-metre telescopes at Siding Springs and 


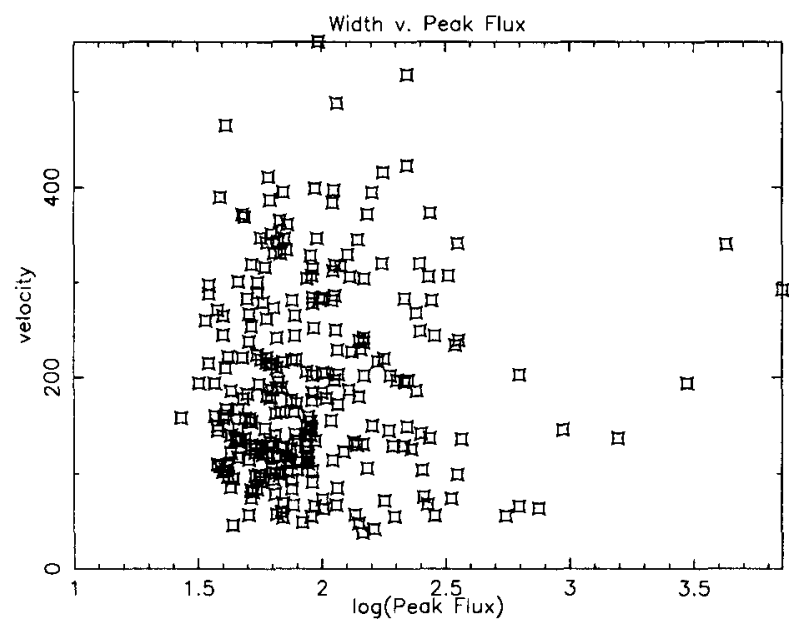

Figure 1. Plot of velocity width against peak intensity for 274 galaxies detected in the southern in the galactic pole region.

Las Campanas. Our data will also be combined with data from other all-sky surveys at different wavelengths, eg. near-IR.

\section{HI Mass Function}

The HI mass function has been determined for two regions of sky which have been fully scanned. The -74 region is an $8^{\circ}$-wide declination band centred on $\delta=-74$. A total of 99 galaxies have been detected in 0.242 steradians of sky or about $4 \%$ of the southern sky. Confused sources have been omitted from the catalogue. Four different people have searched the cubes for galaxies. Figure 1 plots the peak flux of detected galaxies as a function of measured velocity width at $20 \%$ of the peak intensity. A clear cutoff is shown at $v \sim 50 \mathrm{~km} \mathrm{sec}^{-1}$. This cutoff is simply the velocity resolution of the survey. The cutoff in peak intensity is also relatively constant, and may be due to the efficiency of the eye in detecting relatively high peaks against a noisy background. There is a concentration of galaxies in the lower left-hand corner of the plot, suggesting that galaxies in this region will be missed due to the detection limits. High resolution observations from the ATCA are being used to quantify how these observational cutoffs limit the parameter space (inclination, velocity width, peak flux, etc) in which galaxies are observed.

Of the galaxies detected in the -74 region, $14 \%$ are new detections and $36 \%$ have new redshifts. There are a total of $\sim 50 \%$ new HI detections. Only those galaxies with a total flux greater than $4 \mathrm{Jy} \mathrm{km} \mathrm{sec}^{-1}$ are included in the determination of the HI mass function. The $\Sigma_{\frac{1}{V_{M a x}}}$ method is used to determine the mass function in bins of 0.5 in the logarithm of the mass. Distances are 


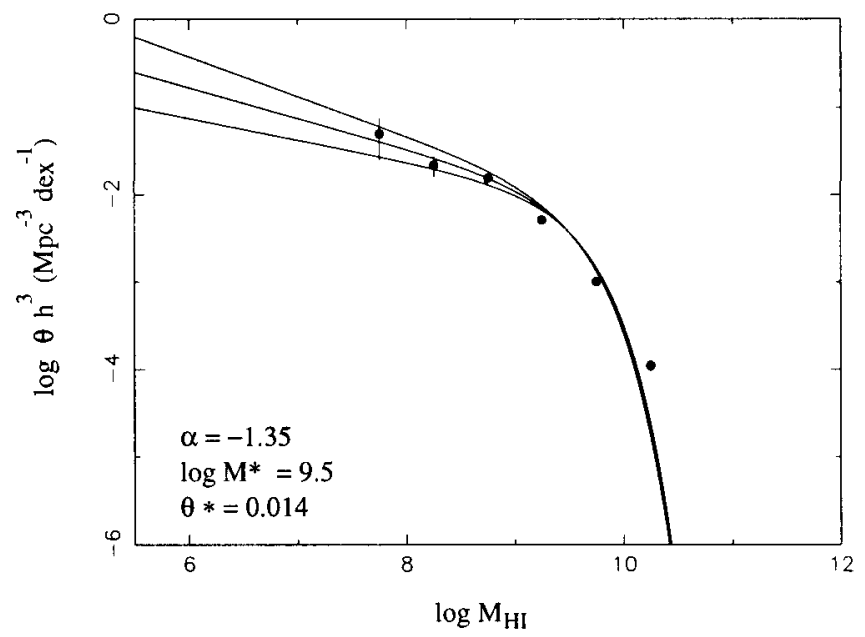

Figure 2. HI mass function 99 galaxies detected in the -74 region.

determined using the Hubble law and a value of $H_{0}=100 \mathrm{~km} \mathrm{sec}^{-1} \mathrm{Mpc}^{-1}$. A Schechter function has been fitted by eye. The functional form is

$$
\theta(x) d x=\theta_{*} x^{\alpha} e^{-x} d x
$$

where $\theta(x)$ is the number of galaxies per decade of mass per $M p c^{3}, x=M_{H I} / M_{*}$ where $M_{*}$ is the characteristic mass defining the knee of the mass function, and $\alpha$ is the low mass slope of the mass function.

The low mass end of the mass function is still poorly determined. There are only a couple of galaxies in the lowest mass bin, and the completeness of the sample has not been determined. In addition, the $\Sigma_{\frac{1}{V_{M a x}}}$ method is sensitive to clustering, whereas a more robust method, such as a maximum likelihood method is not. The fitted values of the Schechter funtction are $\theta_{*}=0.014$ galaxies per $M p c^{3}$ per decade of mass, $\log _{10} M_{*}=9.5$ and $\alpha=-1.35$. The HI mass function is plotted in Figure 2. These values can be compared with other recent determinations. For example, Zwann et al. (1997) find $\alpha=-1.2$ and $\log _{10} M_{*}=9.55$.

Finally, and importantly, so far all of the HI detections have an optical counterpart. Thus we are not detecting HI galaxies which do not contain stars, to the limiting HI surface density of the survey. This is an important result for theories of star formation.

\section{Cen A Group of Galaxies}

The Cen A group of galaxies was extensively surveyed by Côté, Freeman and Quinn (1997). Optical candidates were selected from UK Schmidt plates, and followed up with pointed observations at the Parkes radio telescope. This 

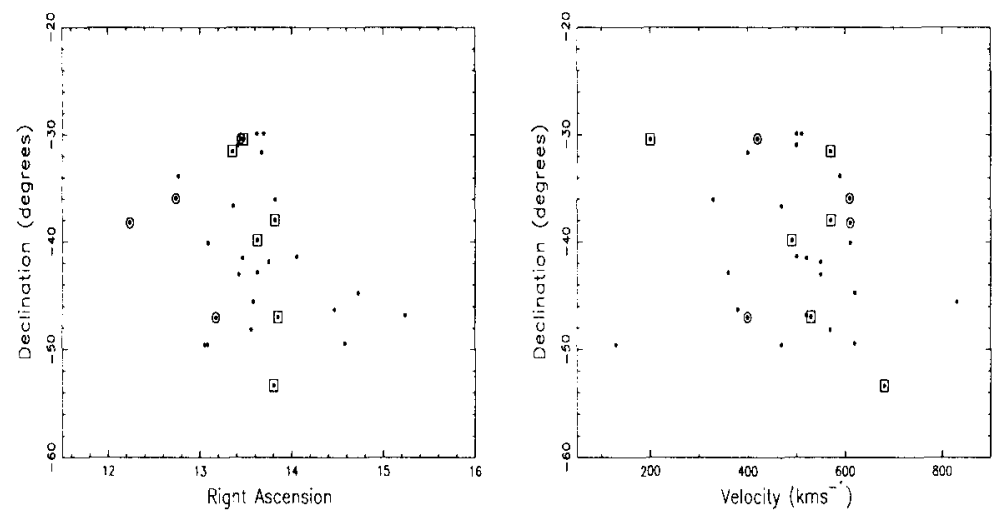

Figure 3. Members in the Cen A group, where the new HIPASS detections are the larger symbols. Both a spatial plot and a plot in velocity space are shown.

work provides a good basis to test the multibeam's survey capabilities. A total of 29 galaxies were detected in the HIPASS data in the veolcity range $200-900 \mathrm{~km} \mathrm{sec}^{-1}$. Of these, 18 had previously been detected, principally by Côté et al. 10 new galaxies were found with fluxes greater than our fiducial cutoff of $4 \mathrm{Jy} \mathrm{km} \mathrm{sec}^{-1}$. Of these 5 were previously catalogued, and 5 were not. Thus the HIPASS observations have increased the number of galaxies detected in the group by $\sim 50 \%$. Figure 3 shows the spatial distribution of galaxies in the Cen A group, with the new members indicated by the larger symbols. Figure 3 also plots the group members as a function of velocity. From this figure it appears that putative members at the extreme ends of the velocity range may not belong to the gravitationally relaxed core of the group. Indeed if traditional cluster member algorithms are applied (Yahil and Vidal 1977), then only those galaxies in the velocity range $300-700 \mathrm{~km} \mathrm{sec}^{-1}$ satisfy the $3 \sigma$ cutoff criterion. A Schechter function can be fitted to the mass distribution for the galaxies in the group, assuming that they are all located at a distance of $3.5 \mathrm{Mpc}$. Values of $\log _{10} M_{*}=9.3$ and $\alpha=1.3$ are obtained.

In addition, the region around all the optically verified members of the group was exhaustively searched by eye, resulting in a detection of ESO 272 G025 with a flux of $1.2 \mathrm{Jy} \mathrm{km} \mathrm{sec}^{-1}$, which is a $\sim 3 \sigma$ detection. Figure 4 shows the HIPASS profile of this galaxy. A blind eye search would not have found this galaxy, however this detection provides a benchmark with which automated finding algorithms can be tested.

\section{Galaxy Formation}

Our preliminary datasets all give a slope for the low mass end of the mass function of $\alpha \sim-1.3$, irrespective of whether we search in the field or in a 


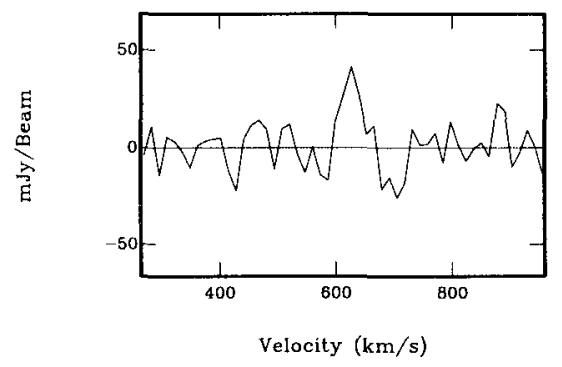

Figure 4. HIPASS profile for ESO272-G025, an optically detected member of the Cen A group. This is a $3 \sigma$ detection.

denser galaxy environment. Improved galaxy detection algorithms may increase the detections at the low mass end and a more sophisticated treatment of the incompleteness will certainly provide a more robust measurement of $\alpha$. Thomas (1997) has predicted the shape of the mass function under a range of cosmological assumptions. In a standard CDM cosmology, if the baryons are located in CDM halos, then using the Press-Schechter formalism, a low mass slope of $\alpha=-1.8$ is calculated for the mass function. The optical galaxy luminosity function has a faint end slope of $\alpha=-1.25$ and this function can be matched to the CDM halo mass function at the knee if a $M / L=15 h M_{\odot} / L \odot$ is assumed for normal galaxies. At the high mass end, the dynamical timescale is greater than the cooling time. Thus we would not expect massive optically luminous galaxies to form. At the low mass end, the CDM halos have a very different distribution from the optically catalogued galaxies. This would result if there was a natural bias of light with respect to the dark matter. In addition, if the low mass end of the HI mass function has a different slope to the faint end of the luminosity function, this would support the idea that the star formation rate in HI halos is not simply a function of total mass. However a full exploration of the $\mathrm{HI}$ parameters of detected galaxies is required before strong conclusions can be drawn from these results.

\section{Dynamics of the Local Group}

With the completion of the first of the five scans, a full mosaic of the southern galactic cap $\left(\delta \lesssim 62^{\circ}\right)$ was generated (Putman et al. 1998). In order to improve the detection of HI structure near the Milky Way, the raw data was reprocessed using a modified bandpass correction (Barnes 1998).

The origin of the Magellanic Stream has remained controversial since its discovery by Matthewson et al. (1977). Currently two models for the stream are considered viable: tidal distortion which predicts a leading arm in conjuction with the trailling Magellanic Stream, and ram pressure stripping by gas in the halo of the Milky Way. Figure 5 shows the full mosaic of the HI distribution. A leading arm is clearly seen stretching from the bridge between the Large 


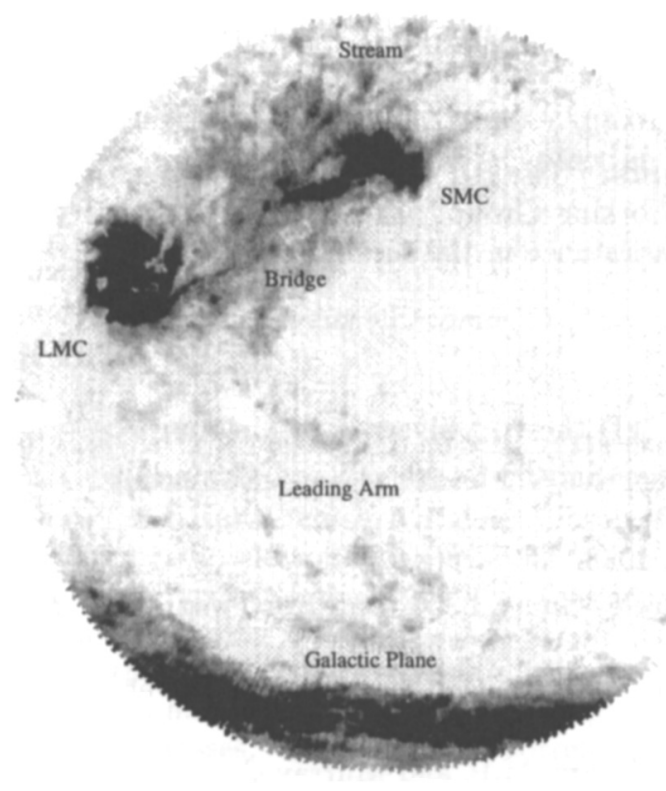

Figure 5. The southern galactic cap as viewed by HIPASS. Different features are labelled.

Magellanic Cloud and the Small Magellanic Cloud towards the Milky Way. This new image is a substantial piece of evidence in favour of the tidal theory for the formation of the Magellanic Stream. This model of the interaction of the LMC/SMC system with the Milky Way supports the idea of Moore et al. (1998) that small disk galaxies are tidally harassed by the larger neighbours in their local environment.

Interestingly, if we also look at the image of the Large Magellanic Cloud in $\mathrm{HI}$, it appears to be a regular spiral galaxy. In optical light, only the bulge is readily visible, and the galaxy is classified as a Irregular. Thus the galaxy morphology based on HI is different from the optical morphology. In addition there is little evidence of tidal distortion in HI distribution in the Large Magellanic Cloud.

\section{Discussion}

The HIPASS survey will be available as a public database, with the first cubes released in late 1998 . So far only about $4 \%$ of the southern sky has been completed, and the data searched albeit by eye. Our planned scientific program is wide-reaching: a HI mass function derived from $>4000$ galaxies with masses $>10^{6} M_{\odot} ;$ a DEEP survey, pushing the limits of detectibility within the constraints of the scanning technique; and a bivariate brightness distribution, based on HI detections with followup optical imaging. The survey will provide a full inventory of the local $\mathrm{HI}$ distribution for regions with $\gtrsim 10^{18} \mathrm{HI} \mathrm{cm}^{-2}-$ an ideal 
laboratory for the study of galaxy formation. Progress on the survey can be found at the website:

http://www.atnf.csiro.au/research/multibeam/multibeam.html.

Acknowledgments. The HIPASS survey is the result of the collaboration of the Multibeam Working Group. The Parkes Observatory staff are thanked for their continued assistance in the survey.

\section{References}

Barnes, D.G. 1998, PhD thesis, University of Melbourne

Barnes, D.G., Staveley-Smith, L., Ye, T. and Oosterloo, T. 1998 ADASS VII (San Franciso)

Henning, P.A. et al, 1998, AJ in press

Henning, P.A., Staveley-Smith,L., Kraan-Korteweg, R.C. and Sadler, E.M., these proceedings

Kilborn, V., de Blok, E., Staveley-Smith, L. and Webster, R.L. 1998, these proceedings

Matthewson, D.S., Schwarz, M.P. and Murray, J.D. 1977 ApJ 217, L5

Moor, B., Lake, G. and Katz, N. 1998 ApJ 495, 139

Putman, M.E. et al. 1998, Nature 394, 752

Staveley-Smith, L. 1997, PASA, 14, 111

Thomas, P.A. 1997 PASA, 14, 25

Yahil, A and Vidal, N.V. 1977 ApJ 214, 347

Zwaan, M., Briggs, F. and Sprayberry, D. ApJ 490, 173 